\title{
A Sensitivity Approach to Force Calculation in Electrostatic MEMS Devices
}

\author{
Min $\mathrm{Li}^{1}$, Dong-Hun Kim ${ }^{2}$, David A. Lowther ${ }^{1}$, and Jan K. Sykulski ${ }^{3}$ \\ ${ }^{1}$ Department of Electrical and Computer Engineering, McGill University, Montreal, QC H3A 2A7, Canada \\ ${ }^{2}$ School of Electrical Engineering and Computer Science, Kyungpook National University, Daegu, 702-701, Korea \\ ${ }^{3}$ School of Electronics and Computer Science, University of Southampton, Southampton SO17 1BJ, U.K.
}

\begin{abstract}
This paper presents a novel method for the computation of force in an electrostatically operated microelectromechanical systems (MEMS) device. The approach is based on continuum design sensitivity analysis (CDSA) and can be used with any analysis system. The method, unlike that of Maxwell stresses, does not require an airgap surrounding the body. The method is applied to the calculation of the tilt angle of a MEMS micromirror, and numerical results are compared with experimental measurements on the real device.
\end{abstract}

Index Terms-Continuum design sensitivity analysis (CDSA), force calculation, microelectromechanical system (MEMS) devices.

\section{INTRODUCTION}

$\mathbf{M}$ ICROELECTROMECHANICAL systems (MEMS)based devices are finding application in a large range of everyday products, from cellular phones to video projectors and optical communications systems and on to sensor systems in automobiles. In almost all of the devices, the electromechanical aspect implies that there is some relative movement between the various components of the device. In the micromirror application, for example, the mirror is attached to a beam which bends under the influence of an electric field generated when a voltage is applied between the mirror and an electrode. The beam acts as a torsion spring and thus resists the rotational motion of the mirror in Fig. 1. The movement of the mirror results in the deflection of a ray of light being reflected by the mirror. Such a structure can be used to optically switch signals whether they are part of an optical communications system or a video projector.

Clearly, the operation of all these devices relies on the generation of force between the various components-this is the mechanism by which the various mechanical effects are created, and-unlike the situation of a motor where the rotational component is disconnected from the static part-in these devices, the goal is to create a stable position where the force due to the electric field is balanced by a mechanical force generated by a spring system. This leads to two observations. The first is that these are all coupled problems of one sort or another and the fundamental operation requires the coupling of electric forces with a structural analysis of some form. The second is that the moving part is solidly connected to the stationary component and thus is not surrounded by an "air" body, i.e., a component on which no force is generated by the electric field.

Thus a critical component in the design and analysis of this class of devices is an accurate prediction of both the global forces acting on a body and their distribution over the surface of the body. The existing methods of force computation fall into two classes. The first is based on virtual work [1] and, in the classical sense, requires field solutions for two configurations

Digital Object Identifier 10.1109/TMAG.2007.916380

Color versions of one or more of the figures in this paper are available online at http://ieeexplore.ieee.org.

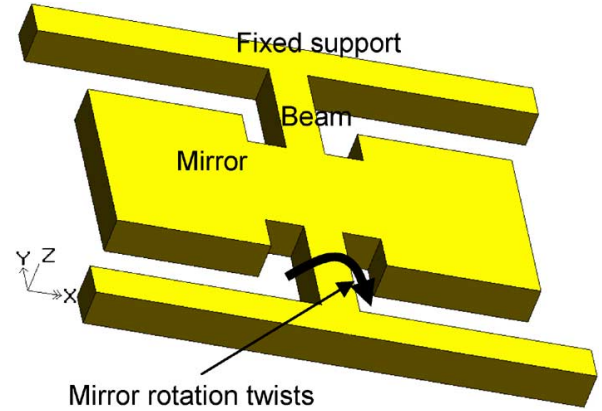

Fig. 1. Basic mirror structure showing torsion beams.

of the device with the component on which the force is desired displaced slightly, in the direction of the required force, between the two solutions. The alternative approach is based on Maxwell stresses [2] and the forces are obtained from an integration of field components around a contour (in 2-D) or surface (in 3-D) enclosing the body. This requires only one field solution but is highly sensitive to the process used to obtain the fields. In general, the fields are based on the derivative of the potential used to solve the problem and this introduces errors. In addition, the contour needs to be defined in an air region surrounding the body. Both of these methods are used widely but require considerable work to produce accurate answers and both have disadvantages which have been well documented in the literature.

In our recent papers [3], [4], a new approach to force calculation for magnetic fields based on continuum design sensitivity analysis (CDSA) has been described. In a sense this is a development of the standard virtual work approach developed in [1] but with a significant advantage that the analysis method used to determine the fields is irrelevant. This approach also has the interesting - and from the practical point of view-a very important property that, unlike the Maxwell Stress method [2], it is not necessary for there to be an air region surrounding the body. In addition, as will be shown below, only a single solution of the field problem is required. The approach provides not only the global force on a body but also the force distribution over its surface.

The intention of this paper is to derive an electrostatic force equation based on CDSA for use with MEMS devices. The approach is then applied to a micromirror system. 


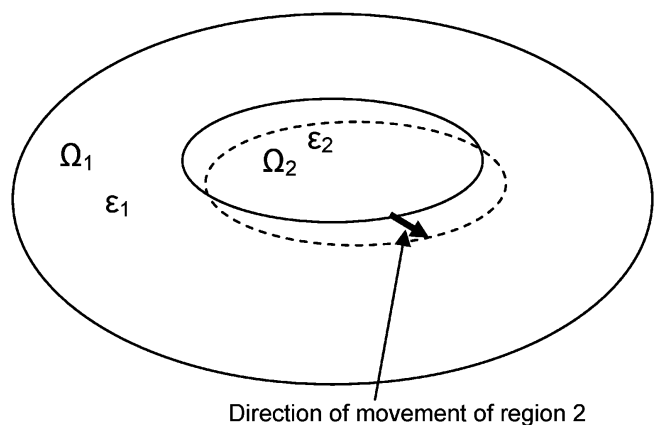

Fig. 2. Virtual movement of body 2.

\section{CDSA-BASEd Force CALCUlation}

In order to derive the energy sensitivity formula, first, an objective function $W$ (the total stored energy in the system) is mathematically expressed as

$$
W=\int_{\Omega} g(\varphi(\mathbf{p})) d \Omega
$$

where $g$ represents an energy function of the electrostatic system, differentiable with respect to the electric scalar potential, $\varphi$, that is an implicit function of the design variable vector p. The derivation of the sensitivity formula to describe the force $(\partial \mathbf{W} / \partial \mathbf{p})$ requires that the variational form of Poisson's equation, referred to as the primary system, is added to (1) using the augmented Lagrangian method

$$
\bar{W}=\int_{\Omega} g(\varphi) d \Omega+\int_{\Omega} \lambda\left\{\varepsilon \nabla^{2} \varphi+\rho\right\} d \Omega
$$

where $\rho$ is electric charge density and $\lambda$ is the Lagrange multiplier and interpreted, in this case, as the adjoint variable.

To obtain an explicit expression for the deformation of the interface boundary between different materials, $\Omega_{1}$ and $\Omega_{2}$, and accordingly for the variation of the permittivity distribution inside the materials, the second integral on the right-hand side of (2) is split into the two regions (Fig. 2).

Then the material derivative on both sides of (2) is taken as

$$
\begin{aligned}
\dot{\bar{W}}= & \int_{\Omega_{1}} g_{\varphi} \cdot \bar{\lambda}_{1} d \Omega \\
& -\int_{\Omega_{1}} \varepsilon_{1}\left\{\nabla \varphi_{1} \cdot \nabla \bar{\varphi}_{1}+\nabla \lambda_{1} \cdot \nabla \bar{\lambda}_{1}\right\} d \Omega \\
& -\int_{\Omega_{2}} \varepsilon_{2}\left\{\nabla \varphi_{2} \cdot \nabla \bar{\varphi}_{2}+\nabla \lambda_{2} \cdot \nabla \bar{\lambda}_{2}\right\} d \Omega \\
& +\int_{\Omega_{1}}\left\{\rho_{1} \bar{\varphi}_{1}\right\} d \Omega+\int_{\Omega_{2}}\left\{\rho_{2} \bar{\varphi}_{2}\right\} d \Omega \\
& +\int_{\gamma}\left\{\varepsilon_{1} \nabla \varphi_{1} \cdot \nabla \lambda_{1}-\varepsilon_{2} \nabla \varphi_{2} \cdot \nabla \lambda_{2}\right\} V_{n} d \gamma \\
& +\int_{\gamma}\left\{\rho_{2} \lambda_{2}-\rho_{1} \lambda_{1}\right\} V_{n} d \gamma
\end{aligned}
$$

where $\mathrm{g}_{\varphi} \equiv \partial \mathrm{g} / \partial \varphi, \bar{\varphi} \equiv(\dot{\lambda}-\nabla \lambda \cdot \mathbf{V}), \bar{\lambda} \equiv(\dot{\varphi}-\nabla \varphi$ $\mathbf{V})$, and $\mathbf{V}$ denotes a design velocity vector, i.e., the direction of boundary movement, and $\gamma$ denotes the part of the interface boundary that is allowed to move. The integrands related to $\bar{\varphi}$ and $\bar{\lambda}$ in (3) vanish because they have the same variational forms as the primary system and the adjoint system, respectively.

Finally, the energy sensitivity formula applicable to electrostatic problems is given by

$$
\begin{array}{r}
\frac{d \bar{W}}{d \mathbf{p}}=\int_{\gamma}\left\{\left(\varepsilon_{1}-\varepsilon_{2}\right)\left[\frac{\varepsilon_{1}}{\varepsilon_{2}}\left(\nabla \varphi_{1}\right)_{n}\left(\nabla \lambda_{1}\right)_{n}+\left(\nabla \varphi_{1}\right)_{t}\left(\nabla \lambda_{1}\right)_{t}\right]\right\} \\
\times V_{n} d \gamma+\int_{\gamma}\left\{\left(\rho_{2}-\rho_{1}\right) \lambda_{1}\right\} V_{n} d \gamma
\end{array}
$$

where the surface integral represents the variation of the stored total electric energy experienced over the interface. When dealing with the objective functions related to the system energy, the dual system consisting of the primary and the adjoint systems is self-adjoint. In other words, the pseudosource of the adjoint system is the same as that of the primary system. Thus $\varphi=\lambda$ and there is no need to solve the adjoint problem, which is obviously of significant benefit in practical computational schemes.

From the energy sensitivity equation (4), the mechanical force $\mathbf{F}$ acting on the interface $\gamma$ between two different electric materials of $\varepsilon_{1}$ and $\varepsilon_{2}$ can be written as

$$
\begin{aligned}
\mathbf{F}=\int_{\gamma} \mathbf{f}_{s} d \gamma \\
=\int_{\gamma}\left\{\frac { 1 } { 2 } ( \varepsilon _ { 1 } - \varepsilon _ { 2 } ) \left[\frac{\varepsilon_{1}}{\varepsilon_{2}}\left(\nabla \varphi_{1}\right)_{n}\left(\nabla \lambda_{1}\right)_{n}\right.\right. \\
\left.\left.+\left(\nabla \varphi_{1}\right)_{t}\left(\nabla \lambda_{1}\right)_{t}\right]\right\} \mathbf{n} d \gamma
\end{aligned}
$$

where $\mathbf{f}_{s}$ is the surface force distribution, and the direction of each surface force is decided by an arbitrary design velocity $\mathbf{V}$. In this case, $\mathbf{V}$ is set to the same direction as a unit normal vector $\mathbf{n}$ outward to $\gamma$. It is also worth repeating here that the surface integration on $\gamma$ does not imply the need for the presence of an air region surrounding the body (or part of it) on which the force is being determined.

The force acting on the surface charge density existing on a conductor is given as

$$
\begin{aligned}
\mathbf{F}_{\rho} & =\int_{\gamma} \mathbf{f}_{s} d \gamma \\
& =\int_{\gamma}\left\{\left(\rho_{2}-\rho_{1}\right) \lambda_{1}\right\} \mathbf{n} d \gamma .
\end{aligned}
$$

\section{MICROMIRROR EXAMPLE}

Fig. 3 shows a plan view (from below) of a simple micromirror structure constructed using the MUMPS process [5] (the mirrors are above the electrodes). All the electrodes and the mirror structures are constructed from polysilicon with a relative permittivity of 11 . The central mirror is connected by a set of torsion springs to an outer gimbal which in turn 


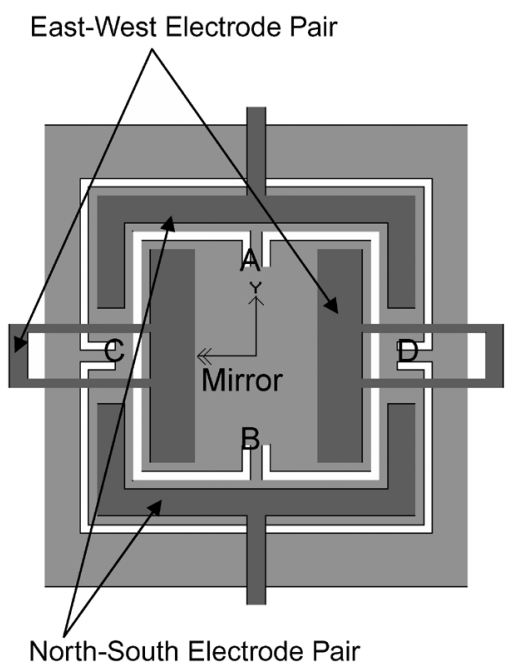

Fig. 3. Basic structure of micromirror device.

is connected to a fixed frame (all shown in light gray in the figure). The micromirror itself is a square of side $130 \mu \mathrm{m}$; the first frame around it has an outer dimension of $190 \mu \mathrm{m}$. The thickness of the mirror layer is $2 \mu \mathrm{m}$, that of the electrodes is $0.5 \mu \mathrm{m}$, and the airgap between the electrodes and the mirror is $1.5 \mu \mathrm{m}$. Beams A and B act as torsion springs for east-west rotation, while $\mathrm{C}$ and $\mathrm{D}$ provide north-south rotation.

The system is energized by two pairs of electrodes under the mirror structure (shown in dark gray in the figure)

While the model is truly 3-D, the first analysis was performed on a 2-D cross-section of the model with the east-west (horizontal) electrode pair excited. The reaction torque due to the bending of the suspension (torsion springs) was computed from a simple analytical model assuming a rectangular cross-section beam. In this case, the mechanical torque is given by

$$
T_{\text {mech }}=K_{\alpha} \alpha
$$

where $\alpha$ is the angle of rotation and $K_{\alpha}$ is the spring constant of the beam given by

$$
K_{\alpha}=2 \frac{G w t^{3}}{3 l}\left[1-\frac{192 t}{w \pi^{5}} \tanh \left(\frac{\pi w}{2 t}\right)\right] .
$$

$G$ is approximately $69 \mathrm{GPa}$ for polysilicon [6] and can be derived from measured values for Young's modulus and the Poisson ratio, $w$ is the width of the beam, $t$ is the thickness of the beam and $l$ is its length.

The determination of the stable position of the mirror was carried out by computing the mechanical restoring torque as a function of the rotation of the mirror. The electrostatic torque was computed using both the new CDSA approach and the internal force calculation algorithm in ElecNet [7] which is based on Maxwell stresses. This calculation was carried out for a range of angular positions of the mirror and the stable position was at an angle at which the mechanical restoring torque balanced the electrostatic torque (Fig. 4). The CDSA calculation was implemented using results generated by the ElecNet finite-element-based code although, as stated before, the force calculation system is independent of the analysis method used.

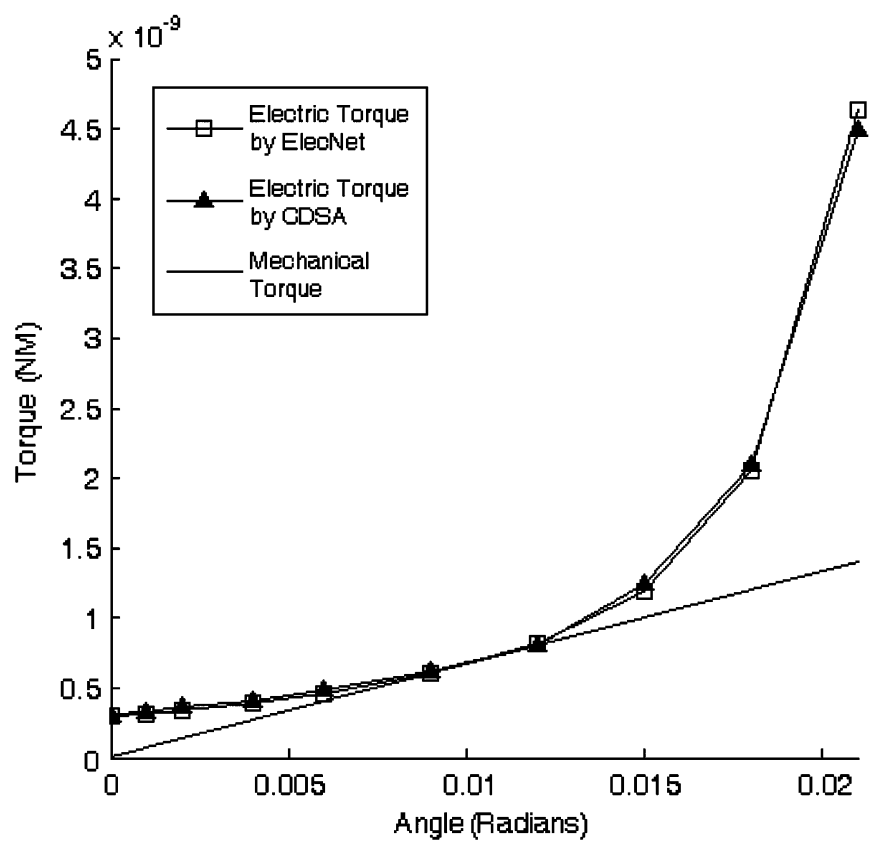

Fig. 4. Torque versus angle for east-west excitation.

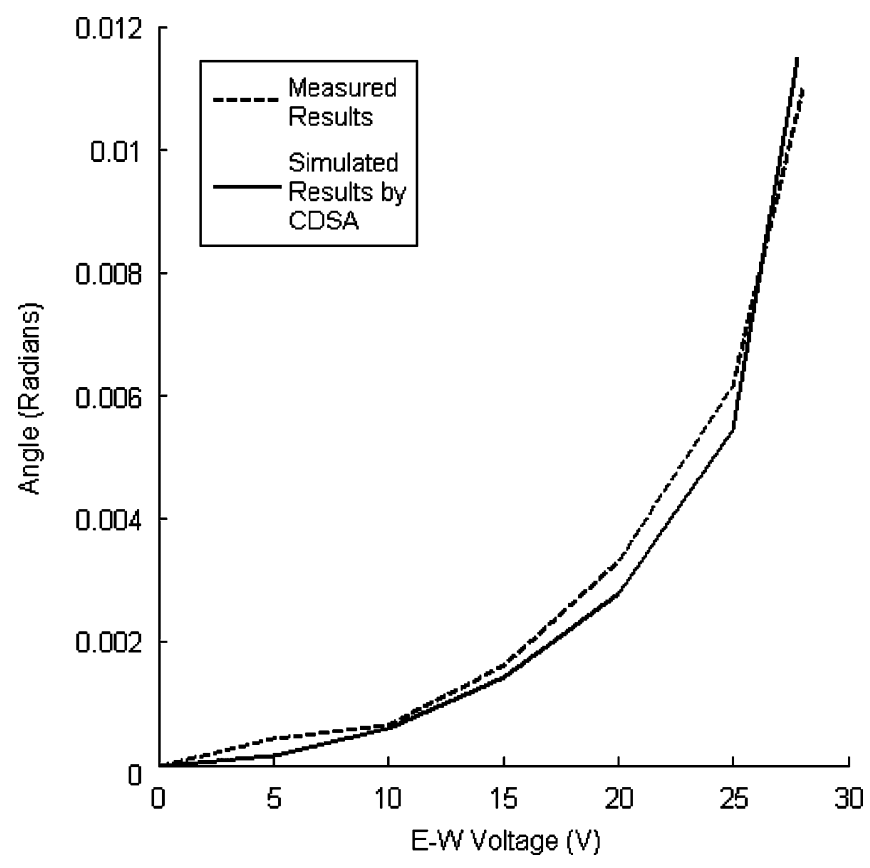

Fig. 5. Tilt angle on north-south axis by exciting the east-west electrodes. Measured versus computed by 2-D analysis.

\section{RESULTS}

Measurements were made on the micromirror in terms of the tilt angle of the mirror versus the applied voltage. Fig. 5 shows the computed tilt angles based on the CDSA force calculation compared with the measured angles for a 2-D analysis.

The same calculations were carried out using a full 3-D analysis model that consists of 58223 third-order tetrahedral elements, and the results are shown in Fig. 6 .

It is interesting to note that the 2-D results appear to be somewhat better than those from the 3-D analysis but this is probably due to several effects cancelling out and is unlikely to be true in 


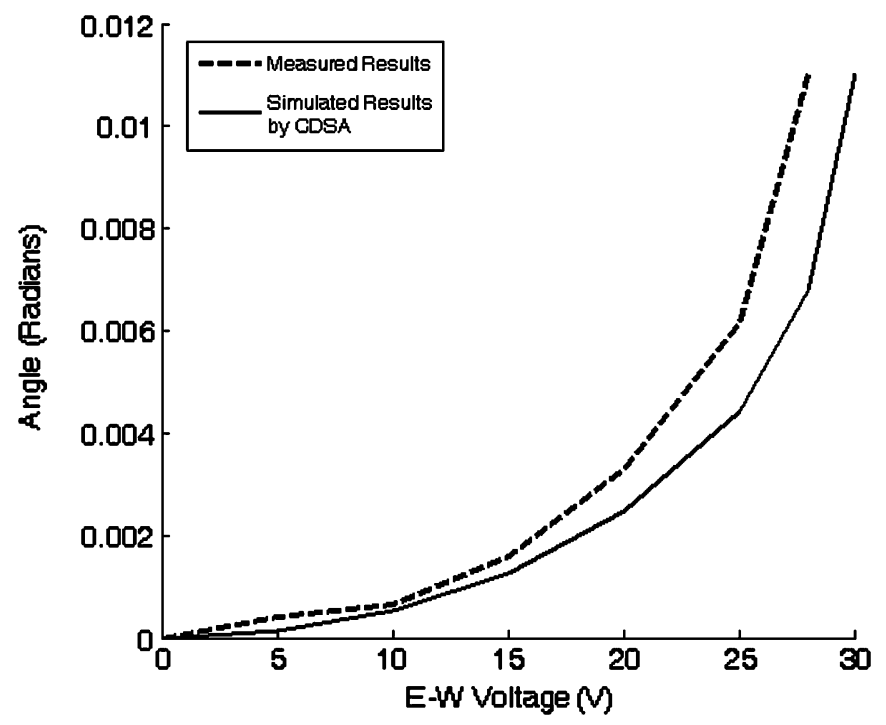

Fig. 6. Tilt angle on north-south axis by exciting the east-west electrodes. Measured versus computed by 3-D analysis.

the general case. The material properties of the polysilicon used in the real device were not measured, instead published values were used, but these have a variation of well over $10 \%$. In addition, it is not known how accurately the final physical device adhered to the dimensions given in this paper. Allowing for these errors, the results of Fig. 6 are probably more in line with what might be expected.

Finally, the comparison between the Maxwell Stress calculation in ElecNet and that from the proposed CDSA approach shown in Fig. 4 indicates that the two approaches produce essentially identical results. This is an indication of the performance of the new method given that the Maxwell stress results used fields computed in air some way away from the mirror surface and also removed the torsion beams (A and B in Fig. 3) in order to surround the mirror with air. The CDSA approach used values over the surface of the mirror and included the beams in the analysis and force calculation. As expected, the beams themselves have little effect on the field solution but it would appear that the CDSA approach is probably more robust than the Maxwell Stress system.

The removal of the requirement of having an air region surrounding the body on which the force distribution is investigated has significant implications, even if the practical system actually has an air gap in the relevant position, as the calculation can be carried out on the surface itself or even "just" inside such a surface. The usual uncertainties associated with the accuracy of field representation in small (thin) gaps are therefore removed. It is also possible to establish the full range of force-displacement characteristics, including the position where different parts of the body are touching.

\section{CONCLUSION}

The paper has described an electrostatic force calculation system which can be applied to a body which is in physical contact with another structure. The effectiveness of the method has been shown through both 2-D and 3-D analyses of a micromirror system and the results compared with experimental measurements on the real device. The advantages of the CDSA approach are clear in that there is no need to worry about an air region surrounding the object subject to the force. In future work, it is intended to add in a more detailed structural analysis and couple this to the surface force distributions to compute the distortion in the mirror surface itself. This is the first time CDSA has been applied to force calculation in an electrostatic system.

\section{ACKNOWLEDGMENT}

The work was supported by the Natural Sciences and Engineering Research Council of Canada.

\section{REFERENCES}

[1] J. L. Coulomb and G. Meunier, "Finite element implementation of virtual work principle to magnetic or electric force and torque computation," IEEE Trans. Magn., vol. 20, no. 5, pp. 1894-1896, Sep. 1984.

[2] C. J. Carpenter, "Surface-integral methods of calculating forces on magnetized iron parts," Inst. Elec. Eng. Monogr., vol. 342, pp. 19-28, 1959.

[3] D.-H. Kim, D. A. Lowther, and J. K. Sykulski, "Efficient force calculations based on continuum sensitivity analysis," IEEE Trans. Magn., vol. 41, no. 5, pp. 1404-1407, May 2005.

[4] D.-H. Kim, D. A. Lowther, and J. K. Sykluski, "Efficient global and local force calculations based on continuum sensitivity analysis," in Proc. Biennial Conf. Electromagnetic Field Computation, Miami, FL, May 2006, p. 158.

[5] D. A. Koester, R. Mahadevan, and K. W. Marcus, SmartMUMPs Design Handbook MEMS Technology Applications Center, MCNC. Research Triangle Park, NC, 1996.

[6] B. Yuan [Online]. Available: http://mail.mems-exchange.org/pipermail/mems-talk/1998-July/001831.html

[7] “ElecNet User's Manual,” Infolytica Corp, 2006.

Manuscript received June 24, 2007. Corresponding author: D. A. Lowther (e-mail: david.lowther@mcgill.ca). 